\title{
Congestion control in vehicular adhoc network: a survey
}

\author{
Shamsul Jamel Elias ${ }^{1}$, Shahirah Mohamed Hatim ${ }^{2}$, Mohamad Yusof Darus ${ }^{3}$, Shapina Abdullah ${ }^{4}$, \\ Jamaluddin Jasmis ${ }^{5}$, R Badlishah Ahmad ${ }^{6}$, Adam Wong Yoon Khang \\ ${ }^{1}$ Universiti Teknologi MARA (UiTM) Kedah, Malaysia \\ ${ }^{2}$ Universiti Teknologi MARA, Tapah Campus, Perak, Malaysia \\ ${ }^{3,4}$ Faculty of Computer and Mathematical Sciences, Universti Teknologi MARA, Shah Alam, Malaysia \\ ${ }^{5}$ Universiti Teknologi MARA (UiTM) Jasin, Melaka, Malaysia \\ ${ }^{6}$ Faculty of Informatics and Computing, Universiti Sultan Zainal Abidin (UniSZA), Malaysia \\ ${ }^{7}$ Fakulti Teknologi Kejuruteraan Elektrik dan Elektronik, Universiti Teknikal Malaysia Melaka, Malaysia
}

\section{Article Info}

Article history:

Received Sep 30, 2018

Revised Dec 22, 2018

Accepted Jan 2, 2019

\section{Keywords:}

Congestion control (CC) VANET

\begin{abstract}
Vehicular adhoc network (VANET) has a significant potential in reducing traffic congestion to provide a stress-free and safer platform for road drivers to travel on the road. However, the current VANET is vulnerable to several challenges which need to be overcome. Congestion control is considered as one of the main challenges in VANET due to the high dynamic topology characteristic. Reliable congestion control (CC) are necessary to provide effectient dissemination of time-critical safety messages in VANET applications; safety and non-safety applications. In this paper, we present the overview on VANET, its application and challenges. We also discuss on the congestion control and provide a brief survey on the congestion control algorithms such as vehicular cloud computing, multiplicative rate decreasing algorithm, multi-objective Tabu search, D-FPAV algorithm and beaconing strategies which have been proposed in order to provide better solutions towards achieving a successful Smart Tranporation System.
\end{abstract}

Copyright $@ 2019$ Institute of Advanced Engineering and Science. All rights reserved.

\section{Corresponding Author:}

Shahirah Mohamed Hatim, Universiti Teknologi MARA, Tapah Campus, Perak, Malaysia. Email: shahirah88@uitm.edu.my

\section{INTRODUCTION}

Road safety has becoming an intense issue in many big cities around the world recently. The number of motorized vehicles is increasing rapidly day to day especially in the urban area, due to one's needs of using transportation for travelling purpose and as the result of the technological advancement of the automotive industry [1]. This situation leads to the problem of traffic congestion caused by the ascending number of circulating vehicles on the road, thus directly affect the emotions, physical and mental comfortability of the road drivers [1]. In today's world of limitless internet access, technologies can be incorporated to reduce the risks of road accidents. In order to increase the safety of road users, drivers should have been provided with the facility to look over potential threats or hazards that can cause harm to them while they are on roads.

In order to overcome the problems, vehicular adhoc network (VANET) is employed by Smart Traffic System to carry out the communication and exchange information between vehicles on the road and the road side units (RSU) [2]-[4]. VANET is a sub-class of Mobile Ad hoc Networks (MANETs) where it used vehicles as mobile nodes [4]-[8]. VANET inherit most of the characteristics of MANETs, but it is equipped with some additional characteristics that make it performed better than MANETs. Dynamic topology, high mobility and high density of the network, are among the features owned by VANET. The 
communication of VANET can be formed either with vehicle to vehicle (V2V) communication or between vehicles to infrastructure (V2I) using IEEE 802.11p radio techology [3],[9].

Due to the quick growth of vehicles density, network might be easily congested. This congestion arises when these networks are flooded by the nodes (vehicles) competing in getting through the networks to obtain information [4], [10]. Thus, the delay and packet loss especially for safety messages will automatically affect the VANET's performance [11]-[13]. Quality of Service (QoS) should be considered to guarantee the reliability and safety of the vehicular communications and to improve the performance of VANET [3]-[4]. A significant need for congestion control strategies is important to improve the VANET performance in delivering safety messages to road users [4], [14]. The objective of congestion control in VANET is to limit the loads on the wireless network to provide better access to the wireless platform through algorithms that reduce the nodes' transmission power.

There are several CC algorithms or methods that has been used to reduce network congestion such as vehicular cloud computing [1], multiplicative rate decreasing algorithm, beaconing strategies, power or rate adaptation, and hybrid algorithm (combination of beacon generation rate and transmission power control algorithm). All the proposed algorithms performed well, but yet suffer from some limitations, such as (a) efficiency issue in communication between equipments and security aspects in the network, (b) incompetent utilization of the connection capacity if the connection is shared by multiple sources and dropout of data packets if congestion happens, (c) could not prevent congestion over the network where it cannot avoid fluctuations in the transmission power or packet generation adjustment process [13], (d) limitation in determining which network in the connection is no longer saturated and status of power value of vehicles connected in the network [13].

In this context, this paper focuses on the overview of VANET and its applications; and discusses on the congestion control (CC) and the algorithms used to overcome network saturation that usually occurs in VANET. The review of the most popular and recently used CC algorithms will be presented to provide a better solution in the area.

\section{VANET OVERVIEW}

As mentioned earlier in the previous section, most the MANETs features are accessible in VANET since it is a sub-class of MANETs. VANET is well-equipped with characteristics such high mobility, infinite energy supply, dynamic topologies etc. [3]-[4]. VANET model consist of the map of streets, highways and roads [3]-[4]. The requirement of identifying the location of vehicles or nodes and their movement path is very important to provide better prediction of future driver decision and prevent the network connection from disconnected or saturated since it is very dependent on the topology change [4]. The VANET applications will determine the requirement of the VANET architecture e.g the usability of devices, strategies, economical and system capabilities, the system performance, and protocol standard) [2], [4], [15]. The simple VANET architecture can see in Figure 1. VANET takes information from the existing system such as Global Positioning System (GPS) and is interconnected wirelessly via embedded On Board Units (OBUs) wireless device [9]. OBUs provide a platform which authorize vehicle to form a short range of wireless ad-hoc networks with the capability of disseminating data and application to vehicular networks [9], [16]-[18].

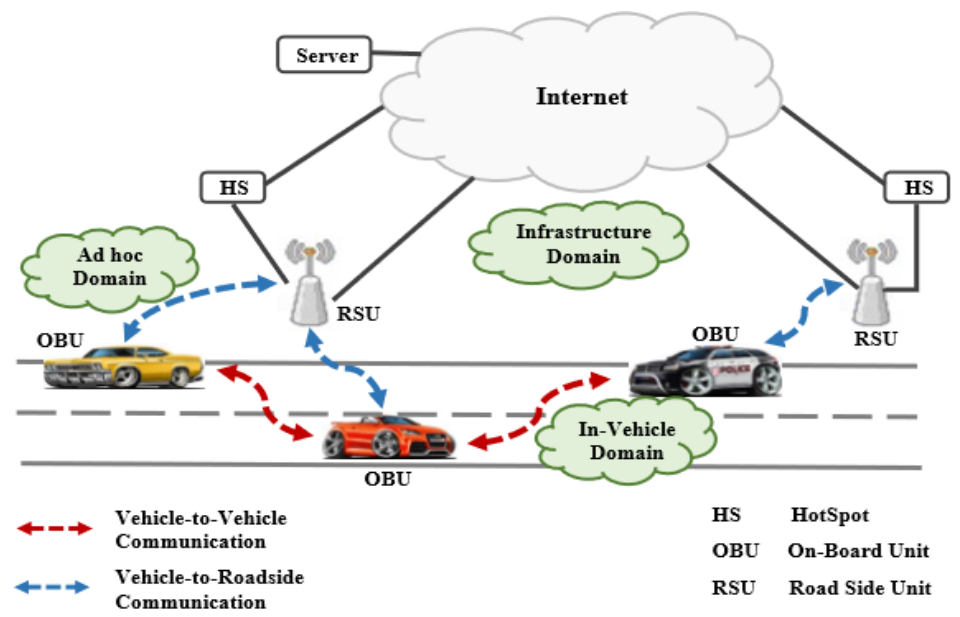

Figure 1. Simple VANET Architecture [4], [10] 


\subsection{VANET Applications}

VANET applications can be classified into two main applications which are safety and non-safety applications [2]-[4], [9], [15], [19]. The objective of safety applications is to prevent road accidents by sending useful information to the road drivers connected in the VANET. Due to the high speed of vehicles on the roads, drivers may not alert with the risky situations. The aim of safety application is to provide lifesaving support to road drivers [2], [4], [9]. For instance, when the safety applications send emergency signals to the vehicles, the road drivers can decrease their speeds towards the accident spot, thus avoiding another accident [4]. Moreover, road drivers can make a turnover or find alternative path to avoid traffic congestion during peak period, which can save time, reduce fuel consumption, and prevent stress [4], [11]. Safety applications use safety messages that can be catogorized into beacon/periodic messages and emergency messages [4], [9]. The beacon messages are oftenly disseminated between the vehicles in the network to transfer or exchange the information about the neighboring vehicles [4], [19]. However, the emergency messages are only sent when an unusual event occurs such as car accidents [2], [4]. Applications such as crash warning and emergency election break lights, used the emergency message [2], [4] whereas information about the vehicle such as position, speed, and route are included in the beacon message [4]. There are some other applications such as the intersection collision warning, low bridge warning, and cooperative collision warning are also included and categorized as beacon messages [3], [4].

The second application of VANET is the non-safety applications. Although it is less important compared to safety applications, it is rather useful in ensuring traffic convenience and efficiency when the drivers are travelling on the road [9]. The purpose of non-safety application is to provide information on the traffic flow, stress-free driving, options for route optimizing and point of interest (POI) [4], [9], [19]. Nonsafety applications of VANET should provide unified internet connectivity to road users since there is a substantial need for people who traveling with vehicles attached to the internet [4]. The example of safety and non-safety VANET applications can see in Table 1.

\begin{tabular}{ll} 
Table 1. The Example of Safety and Non-safety VANET Applications [2],[4],[20] \\
\cline { 2 - 2 } \multicolumn{1}{c}{ Safety Application(s) } & \multicolumn{1}{c}{ Non-safety Application(s) } \\
\hline Traffic violation & Infotainment \\
Curve speed warning & Traffic and route optimization \\
Emergency vehicle warning & Payment services \\
Left turn assist & Point of Interest (POI) \\
Stop sign assist & \\
Co-operative forward collision & \\
Lane change warning & \\
Pre-crash sensing/warning & \\
Emergency brake lights & \\
Collision risk warning & \\
Hazardous location notification & \\
Control loss warning &
\end{tabular}

\subsection{VANET Challenges}

Although VANET is well known with its additional features such as high mobility and high topology rate, yet it raised issues in data routing, data volume, data dissemination, security, and Quality of Service (QoS) [2],[4]. Variety of applications, ranging from traffic safety applications such as traffic violation warning to infotainment applications, such as media downloading requires some sort of supporting VANET technologies thus, leads to several research challenges to be overcome. Among the main challenges identified in VANET are routing protocols [9] anonymity, privacy and security, delay constraints, and congestion control [2],[4].

i) Routing protocols: The application of VANET requires the processing of large volume of data packets. Route stability is crucial in establishing a secure packet delivery [19]. Therefore, a promising routing protocol is needed to ensure secure communications of data broadcasting.

ii) Anonymity, privacy and security: Road drivers should be able to receive information from trusted sources [2]. Thus, privacy and security convey crucial elements that must be satisfied by any VANET applications. It is strictly required in order to ensure verification and privacy of user and data, as well as to provide secure communication since it is dealing with human life. On the other hand, road users might expose to unwanted attempt because it is easily to track vehicles in VANET and if the identifiers e.g Internet Protocol (IP) are never changed in the network. This will make the personal information of the road users such as location and travel path (from home to workplace) can be gathered without difficulty by unknown attackers [2]-[4], [7], [19]. 
iii) Delay constraints: Messages need to be delivered on time to users so that undesirable event such as accidents which could cause the loss of human life, can be prevented. Therefore, a good delay performance should be considered when developing VANET applications to cope with the characteristic of high topology changes [2].

iv) Congestion control: High density of vehicles on the road may caused by the low speed rate of the cars circulating on the road [2],[4]. It is compulsory to offer congestion control approaches in VANET to deal with its characteristics as stated in the previous section. It is very important to prevent packet loss so that the safety message can be dissiminated to the road drivers periodically and safeguard their life.

\section{CONGESTION CONTROL (CC)}

The road users generally use the same resources that are shared over the network thus, can cause network congestion [4]. CC is crucial in order to deliver safety messages to the road users whose are connected to the network without interruption. It is very essential for any of CC algorithms to be able to manage and control the network load to avoid network flood and to ensure the effectiveness of data/message delivery to the end users [4]-[5]. CC algorithms such as vehicular cloud computing [1], multiplicative rate decreasing algorithm [13], beaconing strategies [13], hybrid algorithm etc. Most of the algorithms deal with the adjustment of transmission power control and generation rate control. Studies have been carried out to validate and evaluate the performance of congestion control algorithms [1], [13], [19]. The performance of the $\mathrm{CC}$ algorithms can be evaluated using transmission parameters such as delivery rate, average delay, bit error rate (BER), channel busy ratio (CBR), percentage of successful message reception (PSMR), level of channel congestion (LCC), channel busy fraction (CBF), throughput, average transmission delay (ATD), contention window $(\mathrm{CW})$ etc [13], [19].

\subsection{Existing Congestion Control Algorithms}

Various researches have been performed related to congestion control using different methods. In this section, we briefly survey the studies on existing congestion control algorithms which intended in optimizing the transmission power and the rate of packet generation.

\subsubsection{Vehicular Cloud Computing}

In [1], the authors presented a review on converging VANET with vehicular cloud networks to reduce traffic congestions. It is a new and recent technique where promotes numerous computational facilities which can help in preventing traffic saturation, accidents and driving duration. Vehicular cloud computing creates a cloud where all the services towards the environments are stored in it. Vehicles on the road are the node in the cloud. An expectation of modifying and integrate new algorithm with cloud computing is also pointed by the authors in the review. However, there is no experimental studies has been carried out in the studies, thus creates an argument on the efficiency of the algorithm.

\subsubsection{Multiplicative rate decreasing algorithm}

In [21] which was cited in [13], the authors proposed a new multiplicative rate decreasing algorithm where it reduces the emergency message transmission rate over time. The system can deliver the message especially the emergency warning message over the network in a periodical delay. The algorithm can be said outperform the studies. However, the study only focuses on disseminated emergency warning message for break down car on the road or unexpected car changing lanes.

\subsubsection{Multi-objective Tabu search}

In [4], the author investigated on MO Tabu search to find the nearest solutions of the optimal solution. The author adopted the strategies of tuning the transmission range and rate throughout the studies. The result shows that MO Tabu search can reduce the number of packet loss and delay incurred regardless the density of vehicles circulating on the road compared to another CC algorithm such as D-FPAV. However, the proposed algorithm is restricted to only real-time computations. Moreover, the connected vehicles in the VANET should be equipped with On Board Units (OBU) and GPS to perform the operation.

\subsubsection{D-FPAV algorithm}

In [13], the authors proposed the D-FPAV algorithm where this algorithm worked on modifying the node transmission power to keep the network underload in order to forbid congestion. D-FPAV is executed on a periodic basis and the transmission power rate is adjusted over the time. D-FPAV is using the concept of "min-max" principle by increasing the transmission power rate gradually. However, the study is only managed to reduce $1 \%$ of the overhead down. 


\subsubsection{Beaconing strategies}

In [22] which was cited by authors in [13], the effect on different beaconing strategies were investigated by considering the tracking accuracy parameter. The study aimed to minimize the tracking error rate as well as adopted a beaconing policy to further investigate and compare the tracking error rate with a fixed threshold value. Probability of correctly received beacon by the neighboring vehicles will be increased only if the threshold is exceeded. Although the algorithm helps in decrease the congestion, yet it does not have a total congestion control over the network.

\section{CONCLUSION}

This paper presents an overview on VANET applications and challenges. VANET consists of two major applications; safety application and non-safety application respectively. Safety application includes safety/emergency message that need to be delivered to road users while they are on the road. With the aid of the safety application, human life can be saved. Nevertheless, the non-safety application is equally important as safety application. The non-safety provides additional information to the drivers such as alternative path if unwanted event occurs. However, VANET development contains several challenges such as routing protocol, security issue and the most critical network congestion control. Wireless channel has huge potential to get flooded if it is not being controlled using required mechanism. This may result in interruption to deliver message with safety purpose to the road users when they are travelling.

A survey on the aforementioned CC algorithms shows that all algorithms have its own advantages and limitations. The limitations can be overcome by modifying the algorithms to achieve better result in reducing congestion. The technological advancement in the area makes this a possible move towards achieving a better quality and robust VANET applications.

\section{REFERENCES}

[1] M. S. Talib, et al., "Converging VANET with Vehicular Cloud Networks to reduce the Traffic Congestions: A review," International Journal of Applied Engineering Research, vol/issue: 12(21), pp. 10646-10654, 2017.

[2] G. Karagiannis, et al., "Vehicular Networking: A Survey and Tutorial on Requirements, Architectures, Challenges, Standards and Solutions," IEEE, 2011.

[3] Y. Toor, et al., "Vehicle Ad Hoc Networks: Applications and Related Technical Issues," IEEE Communications Surveys \& Tutorials, vol/issue: 10(3), 2008.

[4] N. Taherkhani, "Congestion Control in Vehicular Ad Hoc Networks," University of Montreal, 2015.

[5] N. B. Truong, et al., "Software Defined Networking-based Vehicular Adhoc Network with Fog Computing," IEEE, 2015.

[6] E. C. Eze, et al., "Advances in Vehicular Ad-Hoc Networks (VANETs): Challenges and Road map for Future Development," 2016.

[7] W. Liang, et al., "Vehicular Ad Hoc Networks: Architectures, Research Issues, Methodologies, Challenges, and Trends," International Journal of Distributed Sensor Networks, vol. 5, 2015.

[8] A. Dua, et al., "QoS-Aware Data Dissemination for Dense Urban Regions in Vehicular Ad Hoc Networks," Mobile Network Application, vol. 20, pp. 773-780, 2015.

[9] S. M. Hatim, et al., "VANETs and Internet of Things (IoT): A Discussion," Indonesian Journal of Electrical Engineering and Computer Science, vol/issue: 12(1), pp. 218-224, 2018.

[10] A. Festag, et al., "NoW-Network on Wheels': Project objectives, technology and achievements," 2008.

[11] T. Ghosh and S. Mitra, "Congestion control by dynamic sharing of bandwidth among vehicles in VANET," Intelligent Systems Design and Applications (ISDA), 2012 12th International Conference on, pp. 291-296, 2012.

[12] M. J. Sattari and R. Md. Noor, "Dynamic D-FPAV congestion control for algorithm for VANETs to rescue human lives," Archives Des Science, vol. 65, 2012.

[13] M. Sepulcre, et al., "Congestion and awareness control in cooperative vehicular systems," Proceedings of the IEEE, vol. 99, pp. 1260-1279, 2011.

[14] N. Taherkhani and S. Pierre, "Congestion control in vehicular ad hoc networks using Meta-heuristic techniques," Proceedings of the second ACM international symposium on Design and analysis of intelligent vehicular networks and applications, pp. 47-54, 2012.

[15] G. Karagiannis, et al., "Vehicular networking: A survey and tutorial on requirements, architectures, challenges, standards and solutions," IEEE Communications Surveys \& Tutorials, vol. 13, pp. 584-616, 2011.

[16] R. Jain, "A Congestion Control System Based on VANET for Small Length Roads," Annals of Emerging Technologies in Computing (AETiC), vol/issue: 2(1), 2018.

[17] Y. D. Mohamad, et al., "Optimizing Congestion Control for NonSafety Messages in VANETs Using Taguchi Method," 2017.

[18] M. Y. Darus and K. A. Bakar. "Congestion Control Framework for Disseminating Safety Messages in Vehicular Ad-Hoc Networks (VANETs)," International Journal of Digital Content Technology and its Applications, vol/issue: 5(2), 2011. 
[19] Elias C. E., et al., "Advances in Vehicular Ad-Hoc Networks (VANETs): Challenges and Road-map for Future Development," International Journal of Automation and Computing, vol/issue: 13(1), pp 1-18, 2016.

[20] H. Hartenstein and K. Laberteaux, "VANET vehicular applications and inter-networking technologies," John Wiley \& Sons, vol. 1, 2009.

[21] X. Yang, et al., "A vehicle-to-vehicle communication protocol for cooperative collision warning," Proceeding First Annual International Conference of Mobile and Ubiquitous System, Network, Services, pp.1-10, 2004.

[22] S. Rezaei, et al., "Tracking the position of neighboring vehicles using wireless communications," Transport. Res. Part C: Emerg. Technol., vol/issue: 18(3), pp. 335-350, 2010. 